\title{
Philosophiques
}

\section{Réponses à mes critiques}

\section{Daniel Laurier}

Volume 30, numéro 2, automne 2003

URI : https://id.erudit.org/iderudit/008651ar

DOI : https://doi.org/10.7202/008651ar

Aller au sommaire du numéro

Éditeur(s)

Société de philosophie du Québec

ISSN

0316-2923 (imprimé)

1492-1391 (numérique)

Découvrir la revue

\section{Citer cet article}

Laurier, D. (2003). Réponses à mes critiques. Philosophiques, 30(2), 421-424.

https://doi.org/10.7202/008651ar

Ce document est protégé par la loi sur le droit d'auteur. L'utilisation des services d'Érudit (y compris la reproduction) est assujettie à sa politique d'utilisation que vous pouvez consulter en ligne.

https://apropos.erudit.org/fr/usagers/politique-dutilisation/ 


\title{
Réponses à mes critiques
}

\author{
DANIEL LAURIER \\ Université de Montréal \\ daniel.laurier@umontreal.ca
}

\section{1. À Montminy}

Je dois admettre que Montminy a parfaitement bien analysé l'argument que je présente en page 144 de mon livre, et qui est censé mener à la conclusion que le naturalisme intentionnel modéré doit être considéré avec pessimisme. En me relisant, (i) je constate que l'argument tel que formulé ne correspond pas exactement à ce que j'avais en tête. Mais (ii) je constate aussi que l'argument que j'avais en tête prête davantage à la controverse que je ne le souhaiterais. Mais qu'à cela ne tienne, puisque (iii) je pense qu'il y a un autre argument qui est beaucoup plus décisif.

(i) Comme le remarque Montminy, j'utilise la prémisse que nul ne peut avoir de pensées conceptuelles à moins d'avoir le concept de rationalité pour conclure qu'une explication de la rationalité dépend d'une explication de la pensée conceptuelle. Et Montminy a raison de souligner que ceci ne découle pas de cela.

Ce que j'avais en tête, ou en tout cas ce que j'aurais dû avoir en tête, c'est plutôt qu'il ne peut pas y avoir de normes de rationalité tout court s'il n'y a pas de créatures capables de les concevoir, de les reconnaître comme telles et de s'y conformer. L'idée est ou aurait dû être que l'existence même des normes de la rationalité dépend de la capacité de les concevoir (et donc que la pensée conceptuelle est en quelque sorte constitutive de la rationalité). C'est ce que je voulais dire, dans ma présentation, quand j'ai souligné que cet argument reposait sur une conception «constructiviste» de la rationalité. L'argument ainsi corrigé permet bien de conclure que l'explication de la pensée conceptuelle et celle de la rationalité ne sont que deux facettes du même problème.

Montminy suggère que mon objection contre le naturalisme intentionnel modéré consiste à montrer qu'une explication naturaliste de la pensée conceptuelle dépend d'une explication naturaliste de la rationalité, et inversement. Cela l'amène à demander pourquoi je n'ai pas envisagé la possibilité d'expliquer simultanément la pensée conceptuelle et la rationalité.

La réponse est que je n'ai nullement ignoré cette possibilité. Au contraire, ce qui m'amène à considérer le naturalisme intentionnel modéré avec pessimisme, c'est précisément qu'on ne voit pas comment on pourrait produire une telle explication "simultanée». Comme je le dis dans le texte, c'est qu'on ne sait pas par quel bout on devrait prendre le problème.

(ii) Ceci dit, je pense que l'argument ainsi corrigé prête flanc à l'objection selon laquelle les normes de la rationalité doivent être considérées 
comme transcendantes, ou en tout cas indépendantes de l'existence de la pensée conceptuelle. Dans cette hypothèse, il n'y aurait plus d'interdépendance entre l'explication de la pensée conceptuelle et celle de la rationalité. Personnellement, je pense que cette hypothèse est inacceptable et que même si elle était vraie, elle ne rendrait pas le naturalisme intentionnel modéré beaucoup plus plausible. Il n'en reste pas moins que c'est une lacune de l'argument tel que présenté d'avoir négligé cette objection.

(iii) Mais tout cela est un peu académique, étant donné que je pense maintenant que l'objection que je soulève dans mon livre contre le naturalisme épistémologique modéré s'applique aussi au naturalisme intentionnel modéré (et que celui-ci n'a donc pas besoin d'un argument spécifique). En deux mots, l'objection repose sur l'idée qu'on ne peut expliquer de notions normatives en termes exclusivement non normatifs. Ce qui revient à dire qu'un naturaliste modéré doit admettre qu'il y a dans la nature des faits irréductiblement normatifs, ce qui trivialise sa position et, de plus, exige qu'il soit capable de montrer que ces faits normatifs contribuent à expliquer des faits non normatifs, ce que je considère comme impossible.

Montminy ne voit pas comment le naturalisme intentionnel radical pourrait conduire à autre chose qu'une sorte de nihilisme intentionnel qui soutiendrait qu'il n'y a pas de contenu du tout. Il remarque que cette position n'est pas à strictement parler auto-réfutante (car il faudrait pour cela qu'elle implique sa propre fausseté, et donc qu'il y ait du contenu), mais qu'elle s'auto-détruit en ce sens que "s'il n'y a pas de signification, alors aussi bien cesser toute activité linguistique... et aller méditer au Bhoutan ».

Je ne pense pas que cette position s'auto-détruit, en tout cas, pas de la manière suggérée par Montminy. Car s'il n'y a pas de signification, alors il n'y a pas de bonne ou de mauvaise chose à faire, puisqu'il n'y a rien qu'on puisse avoir l'intention de faire.

Mais cela n'a pas d'importance, puisque ce que j'appelle le naturalisme radical ne se ramène pas au nihilisme intentionnel ou à l'éliminationnisme. En effet, le naturaliste radical ne soutient pas, comme Montminy le dit, que le contenu n'est pas gouverné par des normes, mais simplement que les normes qui le gouvernent, s'il y a de telles normes, ne sont pas constitutives du contenu. Autrement dit, c'est une position qui considère que le contenu peut s'expliquer en termes non normatifs (par exemple dispositionnels), mais qui n'exclut pas qu'il y ait éventuellement des normes qui gouvernent l'usage de ces contenus. Ces normes auraient un statut comparable aux règles de la conversation de Grice: elles diraient en quelles circonstances il est approprié de produire un énoncé, étant donné que cet énoncé a tel ou tel contenu. Elles seraient, autrement dit, des normes régulatrices et non pas des normes constitutives.

Montminy conclut en suggérant que l'opposition entre le naturalisme modéré et le surnaturalisme pourrait se réduire à la question de savoir si 
les termes normatifs requis pour l'explication du contenu intentionnel font ou non référence à des propriétés naturelles, ou si les principes censés relier les normes aux phénomènes non normatifs sont ou ne sont pas des lois naturelles, et qu'il s'agit là d'une question purement terminologique.

Je crois qu'il se trompe s'il veut dire par là qu'on est libre de répondre à cette question comme on l'entend et que cela n'a aucune conséquence. Car celui qui accepte d'inclure les normes dans la nature a désormais le fardeau de montrer soit que certains phénomènes naturels sont causalement isolés des autres, soit que les faits normatifs ont une efficacité causale. Et dans les deux cas, cela revient, selon moi, à réviser notre concept de nature.

\section{2. À Paternoster}

Je ne trouve pas grand-chose à redire aux commentaires de Paternoster sur la normativité du contenu et les options offertes au naturaliste. Ce qu'il appelle le "quasi-naturalisme" semble se ramener soit à une forme triviale de naturalisme modéré, soit à une forme de surnaturalisme. Il semble d'accord avec moi pour soutenir que la seule forme viable de naturalisme est le naturalisme radical, qui nie la normativité de l'intentionnel.

Paternoster soulève deux questions concernant le type d'approche normativiste que j'évoque brièvement dans le dernier chapitre de mon livre, mais de manière plus développée dans un manuscrit subséquent auquel il fait allusion.

Ma suggestion est qu'on aurait intérêt à explorer la possibilité de produire une explication de la pensée conceptuelle en s'appuyant sur l'idée que certains états intentionnels pourraient avoir des contenus non conceptuels. Paternoster s'inquiète de savoir comment on pourrait envisager de soutenir à la fois que les contenus non conceptuels dérivent des contenus conceptuels et que ceux-ci peuvent s'expliquer en termes de ceux-là, tout en admettant qu'il n'y a pas à strictement parler d'incohérence à le faire'. Je ne pense pas avoir à répondre à cette question, dans la mesure où je n'ai jamais eu l'intention de défendre une telle position. Mon but était simplement de montrer que le fait que Brandom (entre autres) soutienne que l'intentionnalité non conceptuelle est dérivée de l'intentionnalité conceptuelle ne lui interdisait pas pour autant d'admettre que la seconde peut s'expliquer en termes de la première. Je ne pense pas, personnellement, qu'il y ait de bonnes raisons de penser que l'intentionnalité non conceptuelle soit dérivée.

La deuxième question évoquée par Paternoster est celle de savoir si les attitudes déontiques pratiques de Brandom peuvent bien être considérées comme ayant des contenus non conceptuels, ainsi que je le propose. Il

1. Comme je le montre dans une partie du manuscrit mentionné par Paternoster, publié depuis. Il s'agit de «Non-Conceptually Contentful Attitudes in Interpretation ", Sorites, 2001, $\mathrm{n}^{\circ} 3$, p. 6-22. 
suggère d'abord qu'elles pourraient ne pas avoir de contenu du tout, puisqu'elles sont "des habiletés à reconnaître quelqu'un comme étant autorisé ou engagé à produire tel ou tel comportement». Mais je vois mal comment une habileté à reconnaître quelqu'un comme ayant tel ou tel statut pourrait être autre chose qu'une habileté à adopter une attitude ayant un certain contenu.

Plus délicate est la question de savoir si ledit contenu doit être considéré comme conceptuel ou non conceptuel. Paternoster donne quelques raisons de penser que ce contenu est conceptuel. Je ne sais pas ce que valent ces raisons, mais je pense qu'il est effectivement possible de montrer que, dans le contexte de la théorie de Brandom, ces attitudes ne peuvent qu'avoir un contenu conceptuel. Il ne s'ensuit toutefois pas qu'aucune attitude déontique pratique ne peut avoir de contenu non conceptuel. Le problème est de savoir comment caractériser ces attitudes, de telle manière qu'elles puissent jouer un rôle dans une explication de la pensée conceptuelle. Je concède volontiers que «la voie normative pragmatique [...] n'est pas moins difficile que la voie naturaliste non normative». Mais qui a jamais prétendu le contraire? 